\title{
Diffusion of Nonequilibrium Quasiparticles in a Cuprate Superconductor
}

\author{
N. Gedik * and J. Orenstein \\ Physics Department, University of California, Berkeley and \\ Materials Science Division, Lawrence Berkeley National Laboratory, Berkeley, CA 94720 \\ Ruixing Liang, D.A. Bonn, and W.N. Hardy \\ Department of Physics and Astronomy, University of British Columbia, \\ Vancouver, British Columbia, Canada V6T 1Z1
}

\begin{abstract}
We report a transport study of nonequilibrium quasiparticles in a high- $T_{c}$ cuprate superconductor using the transient grating technique. Low-intensity laser excitation (at photon energy $1.5 \mathrm{eV}$ ) was used to introduce a spatially periodic density of quasiparticles into a high-quality untwinned single crystal of $\mathrm{YBa}_{2} \mathrm{Cu}_{3} \mathrm{O}_{6.5}$. Probing the evolution of the initial density through space and time yielded the quasiparticle diffusion coefficient, and both inelastic and elastic scattering rates. The technique reported here is potentially applicable to precision measurement of quasiparticle dynamics, not only in cuprate superconductors, but in other electronic systems as well.

PACS numbers: 74.25.Gz, 78.47.+p
\end{abstract}

Quasiparticles are the elementary excitations of a superconductor, created when a Cooper pair of electrons breaks apart. The dynamic properties of quasiparticles, that is their rates of diffusion, scattering, trapping, and recombination, are critical for applications of conventional superconductors in X-ray detectors [1] and in the manipulation of superconductor-based qubits 2]. In more exotic superconductors, such as the high$T_{c}$ cuprates, a better understanding of quasiparticle dynamics may help to uncover the mechanism for Cooper pairing. A special property of the cuprate superconductors is the d-wave symmetry of the gap function, which leads to an unusual quasiparticle spectrum. The minimum energy for the creation of a quasiparticle depends on the direction of its momentum 3]. It is zero for momenta in the 'nodal' direction, oriented at $45^{\circ}$ relative to the $\mathrm{Cu}-\mathrm{O}$ bond. The most energetically expensive quasiparticles are the 'antinodal' ones, whose momenta are nearly parallel to the bond. The antinodal quasiparticles are the mystery particles of cuprate superconductivity. Because they feel the pairing interaction most strongly, their properties may hold the key to high- $T_{c}$ superconductivity. Unfortunately, their tendency to form strong pairs makes them difficult to study. In thermal equilibrium the population of quasiparticles is overwhelmingly dominated by the low energy nodal ones. As a result, transport measurements performed in equilibrium, such as microwave [4] and thermal [5] conductivity, are insensitive to antinodal quasiparticles.

In this work a transient grating technique was developed and used to probe the transport of nonequilibrium quasiparticles in the high- $T_{c}$ superconductor $\mathrm{YBa}_{2} \mathrm{Cu}_{3} \mathrm{O}_{6.5}$. We find that the diffusion coefficient, $D$, is much smaller than the value obtained in measurements on equilibrium quasiparticles in the same material.

*Electronic address: gedik@socrates.berkeley.edu
The disparity in $D$ suggests that the current experiment probes a population of quasiparticles that are not near the nodes, and are perhaps close to the antinodal regions of momentum space.

The nonequilibrium quasiparticles were introduced using short optical pulses. To probe their propagation, we generated a spatially periodic population by interfering two pulses at the sample surface. The spatial period, $\lambda_{g}$, equals $\lambda / 2 \sin \theta$, where $\lambda$ is the wavelength of the pulse and $2 \theta$ is the angle between the two pump beams. The nonequilibrium quasiparticles cause a change in the index of refraction at the laser frequency [ $[$ ] which is a linear function of their density [7]. As a result, the sinusoidal variation in quasiparticle density creates an index grating which can be detected by the diffraction of a probe pulse.

After creation, the distribution of quasiparticles evolves due to the combined effects of recombination and diffusion. In the process of recombination a pair of quasiparticles jumps back into the Cooper pair condensate with the simultaneous transfer of their creation energy to some other form (e.g. phonons). The amplitude of the grating may also decay as quasiparticle diffusion drives the system towards a spatially homogeneous quasiparticle concentration. The goal of the experiment is to disentangle these effects and measure both the rates of recombination and diffusion.

The transient grating technique has been used successfully in a wide variety of applications, including exciton diffusion, dynamics of biomolecules, propagation of ultrasound, and thermal diffusion [8]. Observing the propagation of superconducting quasiparticles requires the ability to detect the transient grating at extremely dilute concentrations. At the low excitation densities needed to detect their propagation, the quasiparticles produce no more than $10^{-5}$ fractional change in the index of refraction. The diffracted intensity from such a grating would therefore be of order $10^{-10}$ of the incident probe intensity and consequently very difficult to detect. 
Detection of the grating required measurement of the amplitude of the diffracted wave, which is a part in $10^{5}$ of the probe, rather than the intensity. This is accomplished through the use of heterodyne detection [9, 10]. The key element in the success of the heterodyne technique is the diffractive optic (DO) beamsplitter, which creates pairs of pump and probe beams 11, 12]. The DO element in our experimental setup (Fig. 1) is an array of 10 separate $2.5 \mathrm{~mm}$ square phase masks, each with a different grating period, on a fused silica substrate. A beam from a Ti:Sapphire laser $(\lambda=800 \mathrm{~nm}$ and pulse repetition rate $80 \mathrm{MHz}$ ) is split into primary pump and probe beams, which are focused onto one of the phase masks at a small angle with respect to each other. The phase mask splits each of the primary beams by diffraction into the $\mathrm{m}= \pm 1$ orders. (For clarity, only the probe beam paths are shown in Fig. 1).

The interference of the two pump beams creates a spatially varying index of refraction in the sample which reaches a depth $1000 \AA$ below the surface and has half the period of the phase mask. We detect the index variation using an implementation of the heterodyne technique that enables absolute calibration of the phase 13 . Each of the two probe beams is specularly reflected from the surface of the sample, and returns, via the DO, to a Si detector. The two possible round-trip beam paths are shown as solid and dashed lines in Fig. 1. With the use of the DO beamsplitter, detection of the diffracted probe is automatically aligned. The diffracted component of $\mathrm{P} 1$ is precisely colinear with reflected $\mathrm{P} 2$, and vice versa. The experiment is performed by alternately blocking one of the two reflected beams. If reflected $\mathrm{P} 1$ is blocked, then reflected $\mathrm{P} 2$ and diffracted $\mathrm{P} 1$ are mixed in the detector. This measurement by itself is insufficient to extract the wave amplitudes because the relative phase of $\mathrm{P} 1$ and $\mathrm{P} 2$ is undetermined. However, simply blocking reflected P2 instead of $\mathrm{P} 1$ produces a mixed signal with the conjugate phase. Comparing the detector output for the two conjugate beam paths fixes the absolute phase and therefore the wave amplitudes as well.

By adjusting the phase delay of $\mathrm{P} 1$ relative to $\mathrm{P} 2$, the output of the Si photodiode measures either the change of the specular reflection coefficient due to the grating, $R$, or the amplitude of the diffraction efficiency, TG. $R$ is proportional to the spatial average of the quasiparticle concentration, whereas $T G$ is proportional to the component of the quasiparticle concentration at the fundamental period of the grating, $\lambda_{g}$ 14]. In Fig. 2 we plot both $R$ and $T G$ for $\lambda_{g}=2 \mu \mathrm{m}$ as a function of time delay, for several intensities of the excitation pulses, and consequently for a range of initial quasiparticle concentrations. The temperature of the sample was $5 \mathrm{~K}$ and the grating wavevector was oriented along the crystallographic $\mathbf{b}$ axis (parallel to the $\mathrm{Cu}-\mathrm{O}$ chains).

The curves are normalized to their value at time delay zero. The $R$ curves, which decay due to recombination, are nonexponential, and their characteristic rate of decay increases with increasing concentration [7]. The

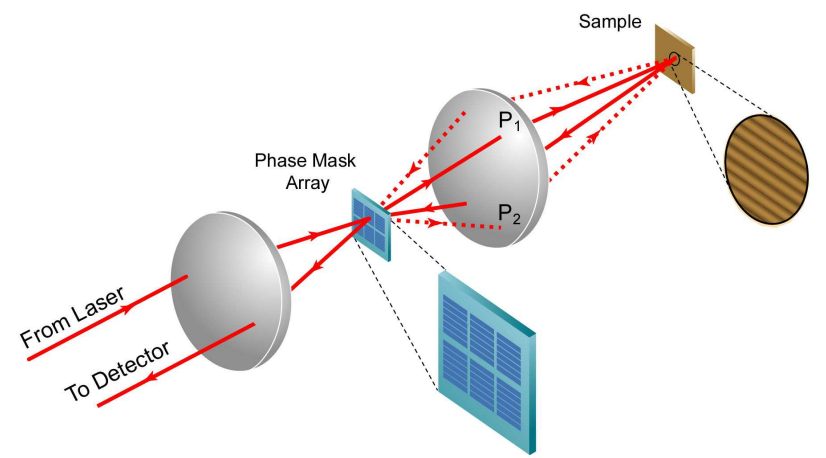

FIG. 1: Illustration of the beam path for heterodyne transient grating detection. Pump and probe beams from the laser are split at the diffractive optic (for clarity only the probe beams are shown). A spherical mirror and plane folding mirror (represented schematically by a lens in the sketch) focus the beams to a single $100 \mu \mathrm{m}$ spot on the sample. After specular reflection and diffraction at the sample surface, the two probe beams are recombined by the diffractive optic and directed to a Si photodiode detector. The wavevector of the quasiparticle density variation is changed, without optical realignment, by translating the diffractive optic so that a different phase mask in the array is inserted in the beam.

increase of recombination rate with density is consistent with the idea that each quasiparticle must encounter another to scatter into the Cooper pair condensate. The corresponding $T G$ curves depend strongly on the pump intensity as well. However the time dependence at each pump intensity is different from $R$ because $T G$ reflects the combined effects of recombination and propagation. In systems where the recombination rate is independent of density, it is relatively straightforward to separate the effects of particle decay and diffusion. If the average concentration decays exponentially with rate $\gamma$, the amplitude of the grating decays with rate $\gamma+D q^{2}$, where $D$ is the diffusion coefficient and $q$ is the wavevector of the grating, $2 \pi / \lambda_{g}$. The ratio $T G / R$, which decays simply as $\exp \left(-D q^{2} t\right)$, isolates the effects of diffusion on the evolution of the particle density.

With this example in mind, we are led to consider $T G / R$ for our data (see Fig. 3). The left, and right, panels show $T G / R$ for grating periods of 2 , and $5 \mu \mathrm{m}$, respectively, for several values of the pump laser intensity. Unlike the example of density-independent recombination, the decay of $T G / R$ is nonexponential and highly dependent on the excitation density. At high intensity $T G / R$ recovers its value at zero time delay, after an initial rapid decrease. $T G / R$ is nearly independent of grating period at high intensity. As intensity decreases, the minimum of $T G / R$ moves systematically towards longer times and the decay approaches a simple exponential. The exponential rate at low pump laser intensity clearly depends strongly on $\lambda_{g}$, as is expected if the grating decays due to diffusion.

The dependence of $T G / R$ on laser intensity and grating period results from an interplay of density-dependent 


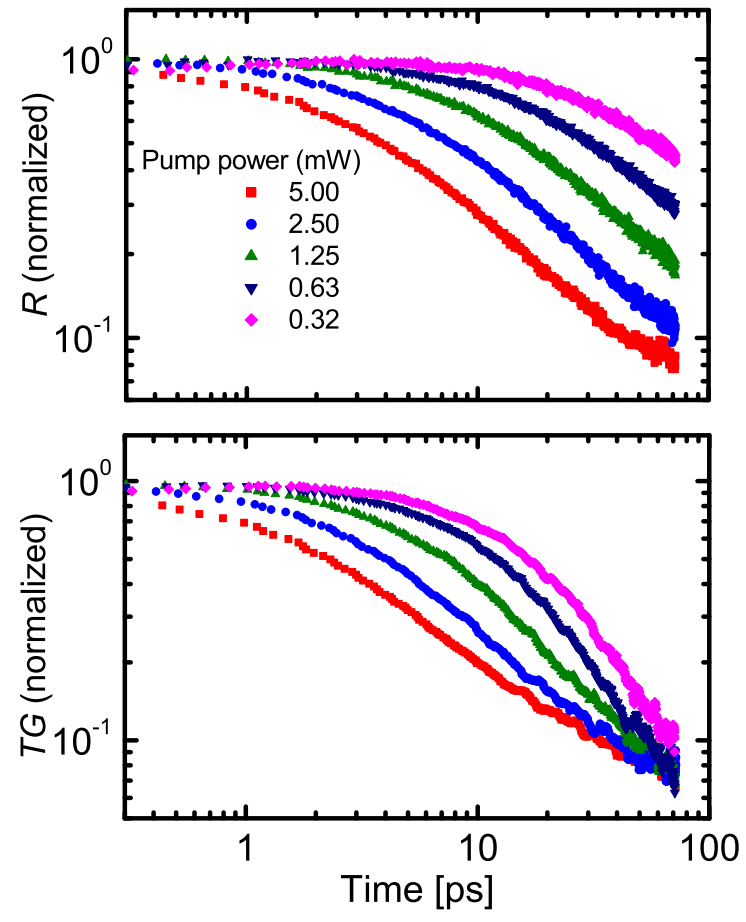

FIG. 2: (top panel) Change in the specular reflection coefficient, $R$, as a function of time delay following creation of the grating, for several values of the pump intensity. $R$ is normalized to unity at time delay zero to illustrate the systematic slowing down of the recombination rate as the excitation density is decreased. (bottom panel) Normalized amplitude of the diffracted probe beam as a function of time delay for the same values of pump intensity used to measure $R$.

recombination, diffusion, and energy transfer. To unravel these effects we have modeled the quasiparticle dynamics by adding a quadratic recombination term to the diffusion equation: $\partial n(x, t) / \partial t=D \partial^{2} n(x, t) / \partial x^{2}-\beta n^{2}(x, t)$ [13]. Here, $n$ is the quasiparticle density and $\beta$ is the recombination coefficient, which is a measure of the inelastic scattering rate [15]. The dynamics predicted by this equation depend on the relative magnitude of $\beta n$ and $D q^{2}$. In the high density regime, $\beta n \gg D q^{2}$, recombination dominates over diffusion. Because the rate is more rapid where $n$ is larger, recombination distorts the sinusoidal grating by flattening the crests. The distortion of the grating profile reduces the component of $n$ at the fundamental grating period faster than its spatial average. This accounts for the initial decrease of $T G / R$. However, if this were the only process $T G / R$ would never bounce back to its initial value, as it is seen to do in Fig. 3.

$T G / R$ recovers in the high-intensity regime because the energy released as the quasiparticles recombine appears in another form, one that also creates a change in the index of refraction. In conventional superconductors the quasiparticle energy is converted to lattice

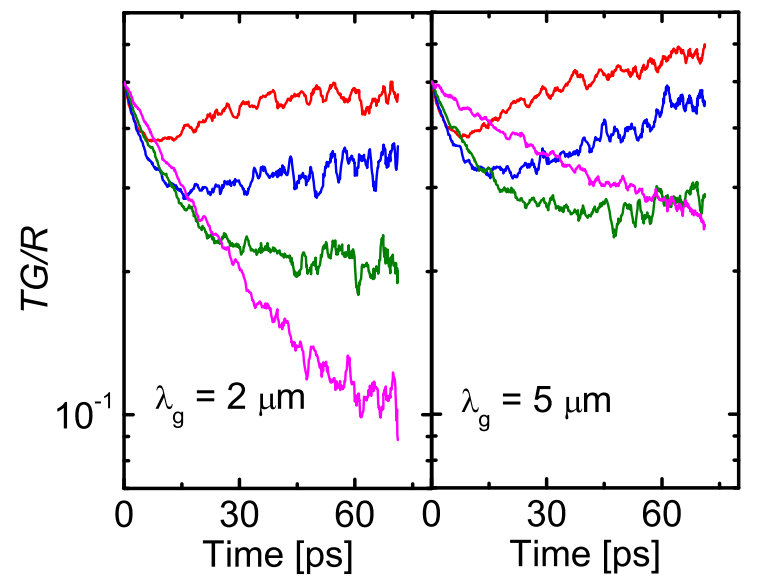

FIG. 3: The ratio $T G / R$ for the same pump intensities as in Fig. 2 (with $\mathrm{P}=0.63 \mathrm{~mW}$ omitted for clarity) for grating period $2 \mu$ (left panel) and $5 \mu$ (right panel). At high excitation density the curves are nearly independent of the period. At low excitation density the grating enters the propagationdominated regime where the decay of $T G / R$ depends strongly on the period of the grating.

vibrational energy, which (in the presence of electronphonon coupling) will change the index. While phonons may play this role in the cuprate superconductors as well, excitations involving the flipping of electron spins may be generated instead when quasiparticles recombine [16]. In either case, the recovery of $T G / R$ follows if this secondary form of energy does not diffuse on the 100 ps time scale. In this case, the original sinusoidal distribution of energy is written into a stationary form of energy before the quasiparticles have had a chance to diffuse. When almost all the energy has been handed off from the quasiparticles to non-propagating modes, the grating recovers its sinusoidal profile and $T G / R$ returns to its value at time zero. The energy remains frozen until the nanosecond time scale, when thermal diffusion becomes significant [17.

The effects of diffusion dominate in the low density regime, $\beta n \ll D q^{2}$, where quasiparticle motion washes out the grating before energy transfer can take place. According to the equation governing $n(x, t), T G / R$ becomes $q$ dependent and intensity independent in this limit. To find the rate of diffusion, we measured $T G / R$ at low power $(\mathrm{P}=0.32 \mathrm{~mW})$ for several grating periods between 2 and $5 \mu \mathrm{m}$. The initial decay rate of $T G / R$ at low power is plotted as a function of $q^{2}$ in Fig. 4. The decay rate depends systematically on the grating period, demonstrating that the dynamics are in the propagation-dominated regime. The rates for the grating oriented along both the $\mathbf{a}$ and $\mathbf{b}$ crystallographic directions are plotted. For both directions the rate is a linear function of $q^{2}$, demonstrating that the quasiparticle propagation is diffusive. From the slope of a linear fit we determine that $D_{a}=20 \mathrm{~cm}^{2} / \mathrm{s}$ 


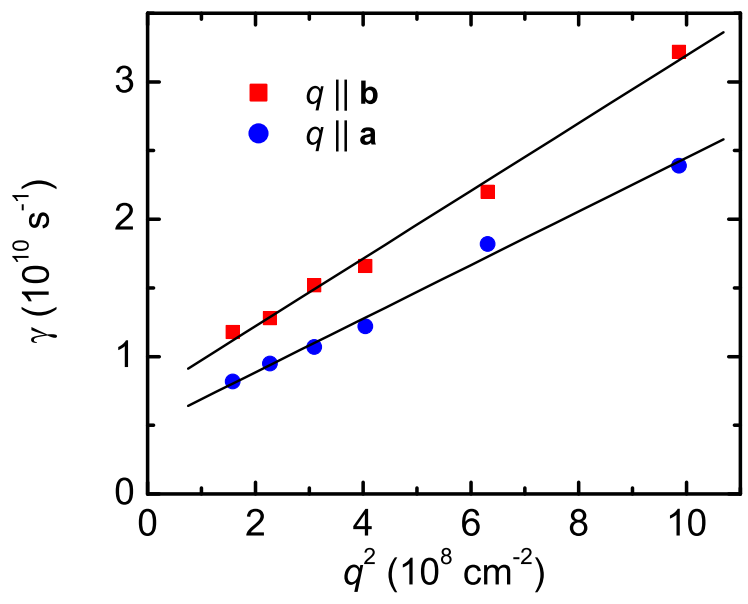

FIG. 4: Initial decay rate of $T G / R$, for the same low intensity as in Fig. 3, plotted as a function of the square of the grating wavevector. Results are shown for two perpendicular orientations of the grating. The linear dependence of the rate on $q^{2}$ demonstrates that the propagation is diffusive. The slopes of linear fits yield diffusion coefficients $D_{a}=20 \mathrm{~cm}^{2} / \mathrm{s}$ and $D_{b}$ $=24 \mathrm{~cm}^{2} / \mathrm{s}$.

and $D_{b}=24 \mathrm{~cm}^{2} / \mathrm{s}$. The intercept as $q$ tends to zero is the decay rate due to recombination.

It is possible to infer the mean free time $\tau$ and mean square velocity $\left\langle v^{2}\right\rangle$ of nonequilibrium quasiparticles from the values of $D$ quoted above. A lower bound on $\tau$ is obtained by inserting the maximum quasiparticle velocity, the Fermi velocity $v_{F}$, into the kinetic formula, $D=<v^{2}>\tau / 2$. The literature value [18] $v_{F}=2 \times 10^{7}$ $\mathrm{cm} / \mathrm{s}$ yields a lower bound of $100 \mathrm{fs}$. An upper bound on $\tau$ of essentially the same value can also be inferred from the experiment. If $\tau$ were significantly longer than 100 fs then quasiparticle propagation would be ballistic on the subpicosecond time scale. However, the time and wavevector dependence of $T G$ proves that quasiparticle motion is diffusive at the earliest times we can resolve, which is $\sim 300$ fs after creation of the grating. Thus the allowed values of $\tau$ and $v$ are narrowly bracketed near 100 fs and $v_{F}$, respectively. Furthermore, the measurements indicate that $\tau$ is determined by elastic, rather than in- elastic, processes. The nonequilibrium particles survive for $\approx 100 \mathrm{ps}$, and therefore scatter $\approx 1000$ times before decaying. This would be impossible if each scattering event resulted in a significant reduction of the quasiparticle's energy.

Equilibrium measurements find (in the same crystal and at the same temperature) that $\tau=20 \mathrm{ps}$ [19], which is 200 times longer than $\tau$ of the nonequilibrium quasiparticles. The contrast suggests that the nonequilibrium quasiparticles are different from those present in thermal equilibrium. The equilibrium particles occupy states which have a small energy $\left(k_{B} T\right)$ relative to the chemical potential, and therefore lie very near the gap nodes. If the nonequilibrium quasiparticles are different, they must occupy higher energy states, perhaps ones closer to the antinodal regions of momentum space. It is possible that the constraints of momentum and energy conservation prevent relaxation of antinodal quasiparticles to the nodal regions. If the particles are antinodal, it is relevant to compare the width of the antinodal quasiparticle peak as measured by photoemission with $\hbar / \tau$ as measured by nonequilibrium transport. The peak width of $14 \mathrm{meV}$ [20] corresponds to $\tau=50 \mathrm{fs}$, which is close to the transport value, particularly considering that photoemission is performed on $\mathrm{Bi}_{2} \mathrm{Sr}_{2} \mathrm{CaCu}_{2} \mathrm{O}_{8}$ rather than $\mathrm{YBa}_{2} \mathrm{Cu}_{3} \mathrm{O}_{6.5}$. The similarity of lifetimes suggests that the peak width in photoemission may be controlled by elastic scattering as well.

The transient grating method reported here promises to be broadly applicable to superconductors, as well as other materials in which there is a gap in the quasiparticle spectrum. The technique works readily in transmission or reflection geometry and therefore can be applied to bulk materials or thin films. The propagation of quasiparticles can be tracked in any system where nonequilibrium excitations generate a change in the index of refraction at the laser wavelength. In conventional superconductors, quasiparticle diffusion can be measured without fabricating trapping layers and junction detectors. In more exotic systems with multiple or anistropic gaps, such as reported in this paper, the transient grating technique can track the propagation of quasiparticles that conventional transport methods cannot detect.

This work was supported by DOE-DE-AC0376SF00098, CIAR, and NSERC.
[1] N.E. Booth and D.J. Goldie, Supercond. Science and Technology 9, 493 (1996).

[2] K.M. Lang, S. Nam, J. Aumentado, C. Urbina, and J.M. Martinis, proceedings of Applied Superconductivity Conference (2002).

[3] Z.X. Shen et al., Phys. Rev. Lett. 70, 1553 (1993).

[4] A. Hosseini et al., Phys. Rev. B 60, 1349 (1999).

[5] Y. Zhang et al., Phys. Rev. Lett. 86, 890 (2001).

[6] J. M. Chwalek, C. Uher, J. F. Whitaker, G. A.
Mourou, and J. A. Agostinelli , Appl. Phys. Lett. 58, 980 (1990).

[7] G.P. Segre et al, Phys. Rev. Lett. 88, 137001 (2002).

[8] H.J. Eichler, P. Gunter, D.W. Pohl, Laser-induced dynamic gratings (Springer-Verlag, Berlin, 1986).

[9] P. Vohringer, N.F. Scherer, J. Phys. Chem. 99, 2684 (1995).

[10] Y.J. Chang. P. Cong. J.D. Simon, J. Phys. Chem 99, 7857 (1995). 
[11] Gregory D. Goodno, Gami Dadusc, R. J. Dwayne Miller, J. Opt. Soc. Am. B 15, 1791 (1998).

[12] A.A. Maznev, K.A. Nelson, T.A. Rogers, Opt. Lett. 23, 1319 (1998).

[13] Details of experimental methods and theoretical modeling are available as supporting material on Science Online at http://science.com/cgi/data/300/5624/1410/DC1/1

[14] I.M. Fishman, C.D. Marshall, and M.D. Fayer, J. Opt. Soc. Am. B 8, 1880 (1991).
[15] S.B. Kaplan et al., Phys. Rev. B 14, 4854 (1976).

[16] S.M. Quinlan, D.J. Scalapino, and N. Bulut, Phys. Rev. B 49, 1470 (1994).

[17] C. D. Marshall, I. M. Fishman, R. C. Dorfman, C. B. Eom and M. D. Fayer, Phys. Rev. B 45, 10009 (1992).

[18] T. Valla et al., Phys. Rev. Lett. 85, 828 (2000).

[19] P.J. Turner et al., cond-mat/0111353

[20] A.V. Fedorov et al., Phys. Rev. Lett. 82,2179 (1999). 